\title{
Modeling and Simulation of Cone Crushers
}

\author{
Andreas Johansson* \\ * Control Engineering Group \\ Lulea University of Technology \\ SE-971 87 Luleå, Sweden \\ Email: andreas.johansson@ltu.se \\ Phone +46920492334
}

\begin{abstract}
A generic structure for dynamic models of cone crushers is presented, in the form of a partial differential equation depending on both time and a spatial variable as well as the size distribution of the particles. Assumptions on the size classification of the flow through the crusher and the velocity of the particles enable modeling the effect of the main manipulated variables, i.e. closed side setting and rotational velocity. It is also shown how the model may be discretized and approximated by an ordinary differential equation. Simulations show that the model exhibits the expected behavior of a cone crusher. Notable is the effect of changing the rotational speed, which suggests that this variable is potentially valuable for improving the control of the crusher.
\end{abstract}

Keywords: Cone crusher, distributed parameter system, partial differential equation

\section{INTRODUCTION}

The cone crusher has received attention for several decades for at least two reasons. Firstly, as all comminution operations, the crushing consumes lots of electric power. Secondly, even quality of the crushing product is important, regardless of whether it is the final product of the plant or going on to further processing. In the latter case, variations in the crusher product may cause problems in e.g. the grinding stage.

In later years, developments in two fields of technology have opened up new possibilities for control of cone crushers. One is the reduction of cost for frequency converters, enabling the use of the rotational velocity as manipulated variable. Furthermore, faster computers and development of new algorithms are making it possible to determine size distributions from image data (Thurley and $\mathrm{Ng}$ (2008)).

Static models of the cone crusher are standard in the literature, see e.g. Lynch (1977) and King (2001). The crusher is then modelled as a single well-mixed crushing zone and the material is distributed into a finite number of size classes. A model where the dynamics is the result of considering several mixing zones is given in Herbst and Oblad (1985). A similar approach is taken here, although we formulate the model as a distributed parameter process, with an infinite number of mixing zones as well as a continuous size distribution Herbst (1979). For the material flow through the cone crusher detailed models are available (Evertsson (1999); Lindqvist and Evertsson (2004)). Here, we will use some of the ideas from these to model the dependence of the rotational speed on both flow and size distribution.

A cone crusher basically consists of a cone-shaped mantle describing a conical motion inside a cone-shaped bowl, see Fig. 1. As the mantle rotates, new rocks are accepted from the feeder into the chamber where they are crushed as they

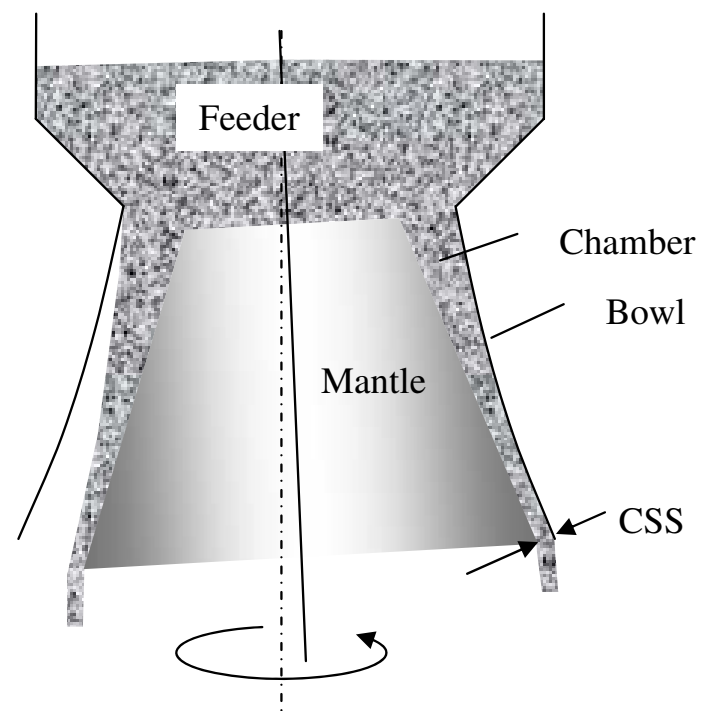

Fig. 1. Vertical cross-section of a cone-crusher

are nipped between mantle and bowl. For each stroke, the rocks move downwards and eventually leave the crusher at the bottom. The closed side setting (CSS) is the smallest distance between mantle and bowl at the outlet and is, together with the rotational speed of the mantle, the main manipulated variables.

The aim of this work is to set up a framework for modeling of cone crushers where different modeling assumptions can be simulated and tested. One important purpose of these models are for closed loop control, which is why we focus on modeling the effect of the manipulated variables and also the dynamical behavior of the size distribution, as we regard this as a quantity that will be possible to measure in real-time. 


\subsection{Notation}

A small interval in a variable $z$ will be denoted $d z$, while infinitesimal intervals, i.e. in integrals and derivatives are signified as $\mathrm{d} z$. The identity matrix is denoted $I$ and a matrix or vector where each element is 1 is denoted 1 . The Kronecker product (also known as tensor or direct product) is denoted by $\otimes$ while $\circ$ signifies the elementwise product (known as Hadamard or Schur product). For a vector $v=\left[\begin{array}{llll}v_{1} & v_{2} & \cdots & v_{n}\end{array}\right], \operatorname{diag}(v)$ is the matrix with the elements of $v$ on the diagonal, while for the matrix $A=\left[\begin{array}{llll}a_{1} & a_{2} & \cdots & a_{n}\end{array}\right]$, the operator vec stacks the columns, i.e. $\operatorname{vec}(A)=\left[\begin{array}{llll}a_{1}^{T} & a_{2}^{T} & \cdots & a_{n}^{T}\end{array}\right]^{T}$. We shall make use of the following properties (Lütkepohl (1996)) of these operations

$$
\begin{aligned}
u \circ v & =\operatorname{diag}(u) v \\
\operatorname{vec}(A \circ B) & =\operatorname{vec}(A) \circ \operatorname{vec}(B) \\
\operatorname{vec}(A B) & =(I \otimes A) \operatorname{vec}(B)=\left(B^{T} \otimes I\right) \operatorname{vec}(A)
\end{aligned}
$$

where $u, v$ are vectors and $A, B$ are matrices of appropriate dimensions.

\section{A DISTRIBUTED PARAMETER MODEL OF A CONE CRUSHER}

By analogy of thermodynamic processes we will set up a material balance of a control volume $\mathcal{C}$ of the crusher. This control volume is not a physical volume but defined as the small level interval $\mathcal{Y}=(y, y+d y)$ in the crusher chamber and a small interval in particle size $\mathcal{D}=(D, D+d D)$. Approximations due to the finite size of this control volume that are made in this section will vanish as we let $d y$ and $d D$ go to zero.

\subsection{A transformed particle density function}

It is customary to use the particle distribution function $P$ when modeling mineral processing operations. This is defined so that $P(D)$ denotes the mass-fraction of particles having a sieve-size less than $D$. The distribution density function is then $p=\mathrm{d} P / \mathrm{d} D$ so that

$$
\int_{D_{2}}^{D_{1}} p(\delta) \mathrm{d} \delta=P\left(D_{1}\right)-P\left(D_{2}\right)
$$

is the fraction of particles with size between $D_{2}$ and $D_{1}$. In practice, discrete versions of the distribution and density functions are almost always used. The discrete particle density function is $p_{i}=P\left(D_{i-1}^{*}\right)-P\left(D_{i}^{*}\right)$ where $D_{i}^{*}$ denote fixed discrete size classes. In this work, we will set up a continuous model of a cone-crusher and it would then appear natural to use the distribution density function $p(D)$. The drawback of this is that $p(D)$ may be unbounded for small $D$, which is the case in the simulation of the cone crusher. Instead, we will defined a logarithmic size scale $\lambda \geq 0$ with base $e$ so that the physical size corresponding to $\lambda$ is $D=D_{0} e^{-\lambda}$ where $D_{0}$ is the largest particle size considered. Then we define a transformed density function as $\chi(\lambda)=D_{0} e^{-\lambda} p\left(D_{0} e^{-\lambda}\right)$. The interval in $\lambda$ corresponding to the size interval $\mathcal{D}$ will be denoted $\Lambda=(\lambda, \lambda-d \lambda)$ where $D=D_{0} e^{-\lambda}$ and $D+d D=D_{0} e^{d \lambda-\lambda}$. The fraction of material in $\mathcal{D}$ may now be calculated as

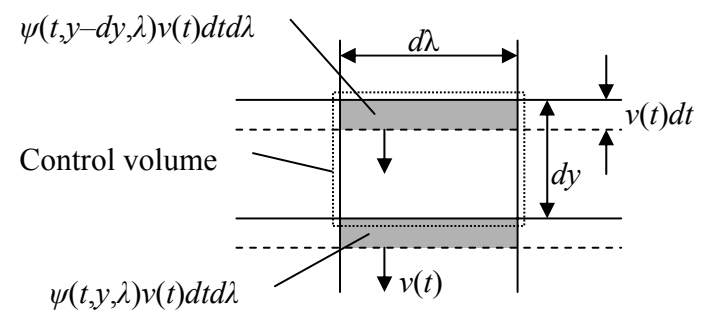

Fig. 2. The control volume

$$
\begin{aligned}
p_{\mathcal{D}} & =\int_{D}^{D+d D} p(\delta) \mathrm{d} \delta=\int_{D_{0} e^{-\lambda}}^{D_{0} e^{d \lambda-\lambda}} p(\delta) \mathrm{d} \delta= \\
& =-\int_{\lambda}^{\lambda-d \lambda} p\left(D_{0} e^{-\lambda}\right) D_{0} e^{-\lambda} \mathrm{d} \lambda= \\
& =\int_{\lambda-d \lambda}^{\lambda} \chi(\lambda) \mathrm{d} \lambda
\end{aligned}
$$

where the third equality was achieved by the variable substitution $\delta=D_{0} e^{-\lambda}$. Assuming a narrow interval $\mathcal{D}$, we may thus approximate $p_{\mathcal{D}} \approx \chi(\lambda) d \lambda$.

The mass of material in a particular size interval $d \lambda$ in a physical volume element $d V$ (not to be confused with the control volume) is obtained as $\chi(\lambda) \rho d V d \lambda$ where $\rho$ is the density of the material. For a cone crusher, it is convenient to use a ring-shaped horizontal cross-section as volume element. The volume of this may be described as $d V=A d y$, where $A(y, t)$ is the cross-section area of the flow at level $y$ and time $t$. To simplify the model formulation, we shall thus define the mass density

$$
x(t, y, \lambda)=\rho A(y, t) \chi(t, y, \lambda)
$$

so that the mass of material in the control volume $\mathcal{C}$ is

$$
x(t, y, \lambda) d y d \lambda
$$

For a flow of velocity $v(t)$ we define the mass flow density as $x(t, y, \lambda) v(t)$ so that the mass flow of particles in size interval $\Lambda$ is $x(t, y, \lambda) v(t) d \lambda$.

\subsection{Classification}

The classification function $c(t, y, \lambda)$ denotes the fraction of material retained for being too large to move downwards. Thus, $1-c(t, y, \lambda)$ is the fraction of material in the control volume that is transported downwards. If the time-average velocity of this transportation is denoted as $v(t)$, then during the time-interval $d t$, the material will have moved $v(t) d t$ meters and the mass in the control volume will have decreased by

$$
\psi(t, y, \lambda) v(t) d t d \lambda
$$

where $\psi(t, y, \lambda)=(1-c(t, y, \lambda)) x(t, y, \lambda)$, see Fig. 2. A similar reasoning concerning the level interval above the control volume indicates an increase of the mass in the control volume of

$$
\begin{aligned}
& \psi(t, y-d y, \lambda) v(t) d t d \lambda \\
\approx & \left(\psi(t, y, \lambda)-\frac{\partial \psi}{\partial y}(t, y, \lambda) d y\right) v(t) d t d \lambda
\end{aligned}
$$

where the approximation was obtained by a 1st order Taylor expansion. The net decrease of the mass density in the control volume due to transportation is thus obtained 
by taking the difference between (4) and (5) and dividing by $d y d \lambda$ which results in

$$
\frac{\partial \psi}{\partial y}(t, y, \lambda) v(t) d t
$$

\subsection{Selection and breakage}

For readability, we suppress the arguments $t$ and $y$ in this subsection. In each nip of the crusher, the fraction $s(\lambda)$ (known as the selection function) of particles of size $\lambda$ will be crushed into smaller sizes. The fraction of broken particles in size interval $\mathcal{D}_{1}=\left(D_{1}, D_{1}+d D_{1}\right)$ that end up in size interval $\mathcal{D}=(D, D+d D)$ after a single breakage event is $B\left(D+d D, d_{1}\right)-B\left(D, d_{1}\right)$ (King (2001)) where $B$ is known as the (cumulative) breakage function and $d_{1}$ is a representative size within size interval $\mathcal{D}_{1}$. Using the logarithmic size scale with $\Lambda_{1}=\left(\lambda_{1}, \lambda_{1}-d \lambda_{1}\right)$ corresponding to $\mathcal{D}_{1}$, this means

$$
\begin{aligned}
& B\left(D_{0} e^{d \lambda-\lambda}, d_{1}\right)-B\left(D_{0} e^{-\lambda}, d_{1}\right) \\
\approx & B\left(D_{0} e^{d \lambda-\lambda}, D_{0} e^{-\lambda_{1}}\right)-B\left(D_{0} e^{-\lambda}, D_{0} e^{-\lambda_{1}}\right) \\
\approx & B^{\prime}\left(D_{0} e^{-\lambda}, D_{0} e^{-\lambda_{1}}\right) D_{0} e^{-\lambda} d \lambda
\end{aligned}
$$

where the first approximation was obtained by replacing $d_{1}$ with the end point $D_{1}$ of the interval and the second approximation is a 1st order Taylor expansion where $B^{\prime}$ denotes the derivative of $B$ with respect to its first argument. By defining $b\left(\lambda, \lambda_{1}\right)=B^{\prime}\left(D_{0} e^{-\lambda}, D_{0} e^{-\lambda_{1}}\right) D_{0} e^{-\lambda}$, the mass of particles in $\mathcal{Y}$ that are broken down from size $\mathcal{D}_{1}$ into $\mathcal{D}$ is

$$
b\left(\lambda, \lambda_{1}\right) s\left(\lambda_{1}\right) x\left(\lambda_{1}\right) d \lambda d \lambda_{1} d y
$$

The total mass of particles in $\mathcal{Y}$ broken down into size interval $\mathcal{D}$ may now be obtained by integrating (7) over all sizes $\lambda_{1}$ from 0 to $\lambda$, i.e.

$$
\int_{0}^{\lambda} b\left(\lambda, \lambda_{1}\right) s\left(\lambda_{1}\right) x\left(\lambda_{1}\right) \mathrm{d} \lambda_{1} d \lambda d y
$$

The increase $\phi(\lambda)$ of the mass density in the control volume $\mathcal{C}$ may thus (according to (3)) be obtained by dividing by the volume $d \lambda d y$ of the interval, i.e. $\phi(\lambda)=(\mathbf{B} s x)(\lambda)$ where the linear operator $\mathbf{B}$ is defined as

$$
(\mathbf{B} z)(\lambda)=\int_{0}^{\lambda} b\left(\lambda, \lambda_{1}\right) z\left(\lambda_{1}\right) \mathrm{d} \lambda_{1}
$$

Also, due to the crushing, the mass $s(\lambda) x(\lambda) d \lambda d y$ will leave the control volume to be broken, i.e. the mass density will decrease by $s(\lambda) x(\lambda)$. The rotational velocity $w$, expressed in revolutions per second (RPS), gives the number of breakage events per second. Thus, the increase of mass density in the control volume during the time interval $d t$ is

$$
w(t)(\phi(\lambda)-s(\lambda) x(\lambda)) d t
$$

It is often assumed that the size distribution of particles after breakage is independent of the initial size, i.e. that the breakage function $B\left(d_{1}, d_{2}\right)$ is in fact a function of the dimensionless parameter $d_{1} / d_{2}$. In this case, we denote $\beta\left(d_{1} / d_{2}\right)=B\left(d_{1}, d_{2}\right)$ and then $B^{\prime}\left(d_{1}, d_{2}\right)=\beta^{\prime}\left(d_{1} / d_{2}\right) / d_{2}$ where $\beta^{\prime}$ denotes the derivative of $\beta$. Thus

$$
\begin{aligned}
b\left(\lambda, \lambda_{1}\right) & =B^{\prime}\left(D_{0} e^{-\lambda}, D_{0} e^{-\lambda_{1}}\right) D_{0} e^{-\lambda} \\
& =\beta^{\prime}\left(D_{0} e^{-\lambda} / D_{0} e^{-\lambda_{1}}\right) D_{0} e^{-\lambda} / D_{0} e^{-\lambda_{1}} \\
& =\beta^{\prime}\left(e^{\lambda_{1}-\lambda}\right) e^{\lambda_{1}-\lambda}
\end{aligned}
$$

Consequently, with $b(\lambda)=\beta^{\prime}\left(e^{-\lambda}\right) e^{-\lambda}$, the breakage operator $\mathbf{B}$ may be expressed as

$$
(\mathbf{B} z)(\lambda)=\int_{0}^{\lambda} b\left(\lambda-\lambda_{1}\right) z\left(\lambda_{1}\right) \mathrm{d} \lambda_{1}
$$

\subsection{The total mass balance}

Combining (8) and (6) gives the mass of particles in the control volume at time $t+d t$ as

$$
\begin{aligned}
x(t+d t, y, \lambda)= & x(t, y, \lambda)-v(t) \frac{\partial \psi}{\partial y}(t, y, \lambda) d t \\
& +w(t)(\phi(t, y, \lambda)-s(t, y, \lambda) x(t, y, \lambda)) d t
\end{aligned}
$$

Finally, letting $d t \rightarrow 0$ and dropping the arguments yields

$$
\frac{\partial x}{\partial t}=w(\phi-s x)-v \frac{\partial \psi}{\partial y}
$$

or, by substituting $\phi$ and $\psi$,

$$
\frac{\partial x}{\partial t}=w(\mathbf{B}(s x)-s x)-v \frac{\partial}{\partial y}((1-c) x)
$$

The boundary value condition to be satisfied concerns the mass density of the feed, i.e. $x(t, 0, \lambda)$. It follows from (2) that this may be expressed as

$$
x(t, 0, \lambda)=\rho A(0, t) \chi_{0}(t, \lambda)
$$

where $\chi_{0}(t, \lambda)=\chi(t, 0, \lambda)$ is the (transformed) density function of the feed.

Equation (9) in combination with the boundary condition (10) is a generic model structure for cone crushers. Specific assumptions of breakage, selection, classification and particle velocity must be made to obtain a model.

The mass flow densities of the feed and the product are

$$
\begin{aligned}
q_{\text {in }}(t, \lambda) & =x(t, 0, \lambda) v(t)(1-c(t, 0, \lambda)) \\
q_{\text {out }}(t, \lambda) & =x(t, Y, \lambda) v(t)(1-c(t, Y, \lambda))
\end{aligned}
$$

and the total mass flows are obtained by integrating these over all $\lambda \geq 0$.

\section{DISCRETIZATION OF THE MODEL}

Models for mineral processing operations are often formulated on a discretized form. The advantage of having a model formulation on a distributed parameter form, such as the partial differential equation (9) is that the modeling problem and the simulation problem are separated. This means that different modeling assumptions can easily be tested while the simulation is conveniently performed by an established mathematical machinery such as the finite element method, for which commercial software is available.

For the simulations in the sequel, we will be using the Matlab Simulink environment, which handles ordinary differential equations. We will therefore demonstrate how a linear PDE in three variables, such as (9), may be approximated by an ODE in a systematic and conceptually simple manner.

The model structure (9) may be written as

$$
\dot{x}=w(\mathbf{B}(s x)-s x)-v\left((1-c) x_{y}^{\prime}-c_{y}^{\prime} x\right)
$$

Let us assume a rectangular grid of points defined by $0=y_{0}<y_{1}<\ldots<y_{N}<Y$ and $0=\lambda_{0}<\lambda_{1}<\ldots<\lambda_{M}$. 
Now, we define matrix-valued functions with the values of $x, x_{y}^{\prime}$, etc., on this grid, i.e.

$$
\begin{aligned}
X(t) & =\left[\begin{array}{ccc}
x\left(t, y_{1}, \lambda_{1}\right) & \cdots & x\left(t, y_{1}, \lambda_{M}\right) \\
\vdots & \ddots & \vdots \\
x\left(t, y_{N}, \lambda_{1}\right) & \cdots & x\left(t, y_{N}, \lambda_{M}\right)
\end{array}\right] \\
X_{y}(t) & =\left[\begin{array}{ccc}
x_{y}^{\prime}\left(t, y_{1}, \lambda_{1}\right) & \cdots & x_{y}^{\prime}\left(t, y_{1}, \lambda_{M}\right) \\
\vdots & \ddots & \vdots \\
x_{y}^{\prime}\left(t, y_{N}, \lambda_{1}\right) & \cdots & x_{y}^{\prime}\left(t, y_{N}, \lambda_{M}\right)
\end{array}\right]
\end{aligned}
$$

and similar definitions for matrices $X_{t}(t), C(t), C_{y}(t), S$, and $\Phi(t)$ using the values of $\dot{x}(t, y, \lambda), c(t, y, \lambda), c_{y}^{\prime}(t, y, \lambda)$, $s(y, \lambda)$, and $\phi(t, y, \lambda)$. We may now express the PDE (11) on the grid as

$$
\begin{aligned}
X_{t}(t)= & w(t)(\Phi(t)-S \circ X(t)) \\
& -v(t)\left((\mathbf{1}-C(t)) \circ X_{y}(t)-C_{y}(t) \circ X(t)\right)
\end{aligned}
$$

The derivative $x_{y}^{\prime}\left(t, y_{i}, \lambda_{j}\right)$ may be approximated by e.g. a first difference

$$
x_{y}^{\prime}\left(t, y_{i}, \lambda_{j}\right) \approx\left(x\left(t, y_{i}, \lambda_{j}\right)-x\left(t, y_{i-1}, \lambda_{j}\right)\right) /\left(y_{i}-y_{i-1}\right)
$$

or some other approximation method, such as the orthogonal collocation method, see e.g. Ding et al. (2007). Either way, we may write

$$
\left[\begin{array}{c}
x_{y}^{\prime}\left(t, y_{1}, \lambda_{j}\right) \\
x_{y}^{y}\left(t, y_{2}, \lambda_{j}\right) \\
\vdots \\
x_{y}^{\prime}\left(t, y_{N}, \lambda_{j}\right)
\end{array}\right] \approx L^{y}\left[\begin{array}{c}
x\left(t, y_{1}, \lambda_{j}\right) \\
x\left(t, y_{2}, \lambda_{j}\right) \\
\vdots \\
x\left(t, y_{N}, \lambda_{j}\right)
\end{array}\right]+L_{0}^{y} x\left(t, y_{0}, \lambda_{j}\right)
$$

for some matrices $L^{y}$ and $L_{0}^{y}$. Thus

$$
X_{y}(t)=L^{y} X(t)+L_{0}^{y} x_{0}(t)^{T}
$$

where $x_{0}(t)^{T}=\left[x\left(t, 0, \lambda_{1}\right) x\left(t, 0, \lambda_{2}\right) \cdots x\left(t, 0, \lambda_{M}\right)\right]$. Vectorizing and using $(1 \mathrm{c})$ gives

$$
\operatorname{vec}\left(X_{y}(t)\right)=\left(I \otimes L^{y}\right) \operatorname{vec}(X(t))+\left(I \otimes L_{0}^{y}\right) x_{0}(t)
$$

The breakage operator may be approximated as

$$
\begin{aligned}
\phi\left(t, y, \lambda_{j}\right) & =\int_{0}^{\lambda_{j}} b\left(\lambda_{j}, \lambda\right) s(t, y, \lambda) x(t, y, \lambda) \mathrm{d} \lambda \\
& \approx \sum_{k=0}^{j-1} b\left(\lambda_{j}, \lambda_{k}\right) s\left(t, y, \lambda_{k}\right) x\left(t, y, \lambda_{k}\right)\left(\lambda_{k+1}-\lambda_{k}\right)
\end{aligned}
$$

On vector form

$$
\left[\begin{array}{c}
\phi\left(t, y_{i}, \lambda_{0}\right) \\
\phi\left(t, y_{i}, \lambda_{1}\right) \\
\vdots \\
\phi\left(t, y_{i}, \lambda_{M}\right)
\end{array}\right] \approx \Gamma\left[\begin{array}{c}
s\left(t, y_{i}, \lambda_{0}\right) x\left(t, y_{i}, \lambda_{0}\right) \\
s\left(t, y_{i}, \lambda_{1}\right) x\left(t, y_{i}, \lambda_{1}\right) \\
\vdots \\
s\left(t, y_{i}, \lambda_{M}\right) x\left(t, y_{i}, \lambda_{M}\right)
\end{array}\right]
$$

where

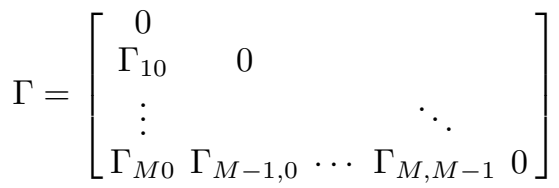

and $\Gamma_{i j}=b\left(\lambda_{i}, \lambda_{j}\right)\left(\lambda_{i}-\lambda_{i-1}\right)$. Thus $\Phi(t)^{T}=\Gamma(S \circ X(t))^{T}$ so that

$$
\begin{aligned}
\operatorname{vec}(\Phi(t)) & =\operatorname{vec}\left((S \circ X(t)) \Gamma^{T}\right) \\
& =(\Gamma \otimes I) \operatorname{vec}(S) \circ \operatorname{vec}(X(t))
\end{aligned}
$$

using (1b) and (1c). Vectorizing (12) gives

$$
\begin{aligned}
\operatorname{vec}\left(X_{t}(t)\right)= & w(t)(\operatorname{vec}(\Phi(t))-\operatorname{vec}(S) \circ \operatorname{vec}(X(t))) \\
& -v(t)\left(\operatorname{vec}(\mathbf{1}-C(t)) \circ X_{y}(t)\right. \\
& \left.-\operatorname{vec}\left(C_{y}(t)\right) \circ \operatorname{vec}(X(t))\right)
\end{aligned}
$$

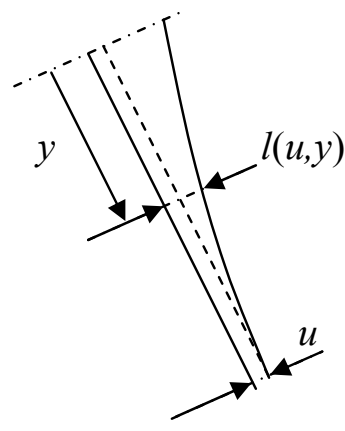

Fig. 3. The chamber thickness $l(u, y)$ in the nip phase. The dashed line marks the chamber profile, i.e. chamber thickness for $u=0$.

Finally, defining

$$
\begin{aligned}
z(t) & =\operatorname{vec}(X(t)) & \bar{C}(t) & =\operatorname{diag}(\operatorname{vec}(C(t))) \\
\bar{S} & =\operatorname{diag}(\operatorname{vec}(S)) & \bar{C}_{y}(t) & =\operatorname{diag}\left(\operatorname{vec}\left(C_{y}(t)\right)\right)
\end{aligned}
$$

and using (1a) results in

$$
\begin{aligned}
\dot{z}(t)= & w(t)((\Gamma \otimes I)-I) \bar{S} z(t) \\
& -v(t)\left((I-\bar{C}(t))\left(I \otimes L^{y}\right)-\bar{C}_{y}(t)\right) z(t) \\
& -v(t)(I-\bar{C}(t))\left(I \otimes L_{0}^{y}\right) x_{0}(t)
\end{aligned}
$$

which is a linear, time-varying, finite-dimensional system, readily implemented in Matlab Simulink.

\section{SIMULATION ASSUMPTIONS}

\subsection{Classification}

A typical form of the classification function is (King (2001))

$$
c^{d}(d)= \begin{cases}1-\left(\frac{d-d_{2}}{d_{1}-d_{2}}\right)^{n}, & d_{1} \leq d<d_{2} \\ 0, & d<d_{1} \\ 1, & d \geq d_{2}\end{cases}
$$

where $d_{1}=\alpha_{1} U$ and $d_{2}=\alpha_{2} U+d^{*}$ and where $U$ is the closed side setting while $n, \alpha_{1}, \alpha_{2}, d^{*}$ are parameters.

Here, we shall use this classification function for each level interval in the crusher with $U$ replaced by the chamber thickness at nip at this level. This chamber thickness depends on the closed side setting $u$ and is denoted $l(u, y)$, see Fig. 3. Thus

$$
c(t, y, \lambda)= \begin{cases}c_{0}(t, y, \lambda), & d_{1}(t, y) \leq D_{0} e^{-\lambda}<d_{2}(t, y) \\ 0, & D_{0} e^{-\lambda}<d_{1}(t, y) \\ 1, & D_{0} e^{-\lambda} \geq d_{2}(t, y)\end{cases}
$$

where

$$
\begin{aligned}
c_{0}(t, y, \lambda) & =1-\left(\frac{D_{0} e^{-\lambda}-d_{2}(t, y)}{d_{1}(t, y)-d_{2}(t, y)}\right)^{n} \\
d_{1}(t, y) & =\alpha_{1} l(u(t), y) \\
d_{2}(t, y) & =\alpha_{2} l(u(t), y)+d^{*}
\end{aligned}
$$

The chamber thickness at nip $l(u, y)$ is assumed to depend linearly on the closed side setting as $l(u, y)=l_{0}(y)+u$ where $l_{0}(y)$ is the chamber profile, defined as the chamber thickness when the closed side setting is $u=0$.

\subsection{Selection and breakage}

A selection function of the form $s_{d}(d)=k d^{a}$ is often used (Kapur (1971)). Then $s(\lambda)=s_{d}\left(D_{0} e^{-\lambda}\right)=k D_{0}^{a} e^{-a \lambda}$. We 
shall therefore assume a selection function of the form $s(\lambda)=s_{0} e^{-a \lambda}$.

A breakage function frequently used for crushers is (King (2001))

$$
B\left(d_{1}, d_{2}\right)=K\left(d_{1} / d_{2}\right)^{n_{1}}+(1-K)\left(d_{1} / d_{2}\right)^{n_{2}}
$$

Then $\beta^{\prime}(\gamma)=K n_{1} \gamma^{n_{1}-1}+(1-K) n_{2} \gamma^{n_{2}-1}$ and $b(\lambda)=$ $K n_{1} e^{-\lambda n_{1}}+(1-K) n_{2} e^{-\lambda n_{2}}$. It may be noted that the breakage operator with this choice of $B$ is nothing but convolution by a sum of two exponential functions which makes it possible to express the breakage operator as two ordinary 1st order differential equations.

\subsection{Particle velocity}

The particle velocity is believed to be mainly affected by the material level in the crusher feeder (Herbst and Oblad (1985)) and the rotational velocity (Evertsson (1999)). For simplicity, we shall here consider only the effect from the latter, i.e. by assuming a constant feeder level. Although more advanced flow models are available (Lindqvist and Evertsson (2004)) we shall use the simple assumption from Evertsson (1999) that the particles are held still during the nipping phase of the stroke and that they fall freely during the rest (e.g. the fraction $\eta$ ) of the stroke. Thus for each stroke, taking time $T_{0}$ to complete, the particles fall freely for $T=\eta T_{0}$ seconds, resulting in a distance of $g T^{2} / 2=g \eta^{2} T_{0}^{2} / 2$. The mean speed of the falling is thus $v=g T^{2} / 2 T_{0}=g \eta^{2} T_{0} / 2$. Since $T_{0}=1 / w$, we shall assume $v(t)=g \eta^{2} / 2 w(t)$.

\subsection{Feed size distribution}

A truncated Rosin-Rammler distribution (King (2001)) of the feed is assumed. This is defined by the particle distribution function

$$
P(D)= \begin{cases}1-e^{-\left(\eta / \eta_{63}\right)^{\alpha}}, & D \leq D_{0} \\ 1, & D>D_{0}\end{cases}
$$

where $\eta=D /\left(D_{0}-D\right)$ and $\eta_{63}=D_{63} /\left(D_{0}-D_{63}\right)$ while $D_{63}$ and $\alpha$ are parameters, both of which may be regarded as functions of time. The corresponding transformed particle density function is then $\chi_{0}(t, \lambda)=D_{0} e^{-\lambda} p\left(D_{0} e^{-\lambda}\right)$ where $p(D)=\mathrm{d} P(D) / \mathrm{d} D$.

\section{SIMULATION RESULTS}

In our simulations, we have found that the orthogonal collocation method gives oscillative results and we have therefore chosen to demonstrate the results of using an ordinary Euler approximation of the derivative. This means

$$
L_{y}=\left[\begin{array}{ccccc}
\frac{1}{y_{1}-y_{0}} & 0 & \cdots & 0 & 0 \\
\frac{1}{y_{1}-y_{2}} & \frac{1}{y_{2}-y_{1}} & & 0 & 0 \\
0 & 0 & \frac{1}{y_{N-1}-y_{N-2}} & 0 \\
0 & 0 & & \frac{1}{y_{N-1}-y_{N}} & \frac{1}{y_{N}-y_{N-1}}
\end{array}\right]
$$

and $L_{0}^{y}=\left[\begin{array}{llll}\frac{1}{y_{0}-y_{1}} & 0 & \cdots & 0\end{array}\right]^{T}$. The grid has been chosen as $y_{i}=i Y / N$ and $\lambda_{i}=i \lambda_{M} / M$ where $N=6, M=23$, and $\lambda_{M}=6.9$.
The model contains several parameters. The values that are used for these are believed to be realistic although not taken from any existing crusher. For the physical dimensions of the crusher we have chosen the length to $Y=1.2 \mathrm{~m}$ and the chamber profile to be linear as $l_{0}(y)=$ $(1-y / Y) l_{0}(0)$ where $l_{0}(0)=0.15 \mathrm{~m}$. The cross-section area of the inflow is chosen to $A(0, t)=0.3 \mathrm{~m}^{2}$ and the density $\rho=1600 \mathrm{~kg} / \mathrm{m}^{2}$. Most of the parameters regarding breakage and classification have been taken from suggested intervals in King (2001). Thus $n_{1}=0.45, n_{2}=3.2$, $\alpha_{1}=0.6, \alpha_{2}=2, n=2$, and $d^{*}=0$. For $K$ and the selection parameters $s_{0}$ and $a$, no suggestions have been found. The values $K=0, s_{0}=0.1$, and $a=0.1$ have been chosen. The time-fraction for free falling $\eta$ is chosen to 0.5 in our simulations. The inflow parameter $\alpha$ is chosen constant as 1.2 .

To see how the model reacts to changes in the inflow size distribution and the manipulated inputs, the inflow parameter $D_{63}$, the rotational velocity $w$, and the closed side setting $u$ have been changed step-wise according to Fig. 4 (top). The middle parts of the figure shows the mass flow densities $q_{\text {in }}$ and $q_{\text {out }}$ respectively, while the lower part shows the total inflow and outflow, i.e. integrated over all size fractions.

In Fig. 5, the size distribution of feed and product is shown at time instants when the flow is stable (marked by vertical, white lines in Fig. 4 (middle)). As expected, the decrease in closed side setting results in a shift of the size distribution towards smaller sizes. More interestingly, an increase in rotational velocity gives decreased material flow as well as dispersion of the distribution towards smaller sizes. This behavior is also expected but has not before been modelled from first principles, as far as we know.

Another expected behavior is that there should be a rotational speed where the material flow is maximized and that decreasing the rotational speed further will give decreased flow as a result of choking the crusher with unbroken material. This behavior is not obtained in the simulations. Instead, very low rotational speeds result in an unrealistic build-up of material in the crusher. Obtaining a flow decrease would require the choking to be modelled, which may be accomplished by modifying the classification function so that less material is transported downwards when the mass density is large. Obviously, letting $c$ depend on $x$ introduces a nonlinearity in the model.

From the perspective of control design, the difference in the reactions of the product on the two manipulated variables can be noted. This suggests that a multivariable control strategy using both the rotational velocity and the closed side setting has the potential to improve the control of the size distribution but also reduce the energy requirement, the latter since the rotational velocity has a significant influence on the power draw. As a simple example of control strategy, the rotational velocity could be used for obtaining a suitable narrowness of the size distribution while the closed side setting is used to shift the size distribution. 

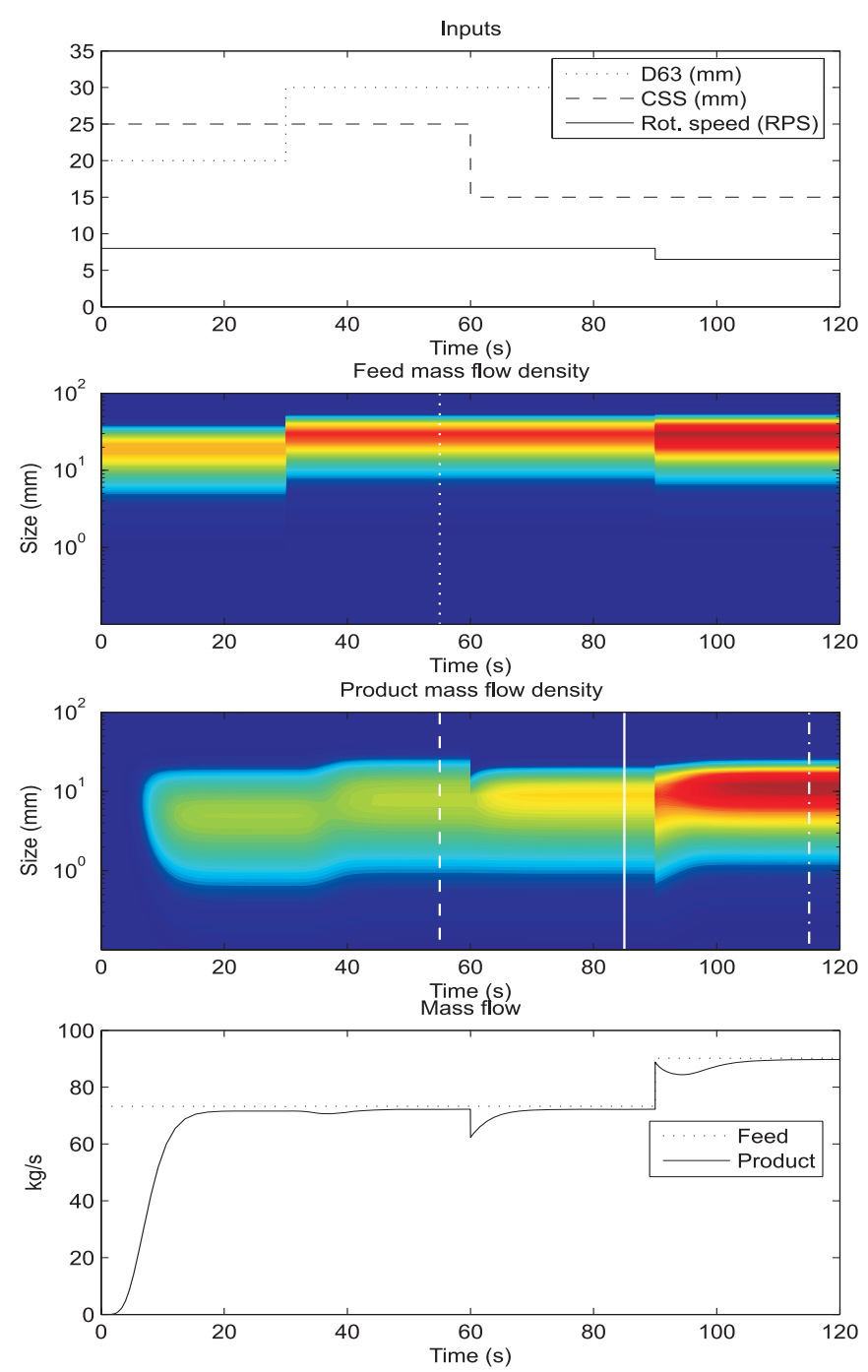

Fig. 4. Simulation of the generic cone crusher model (9) using the assumptions in Section 4 and the parameter values in Section 5

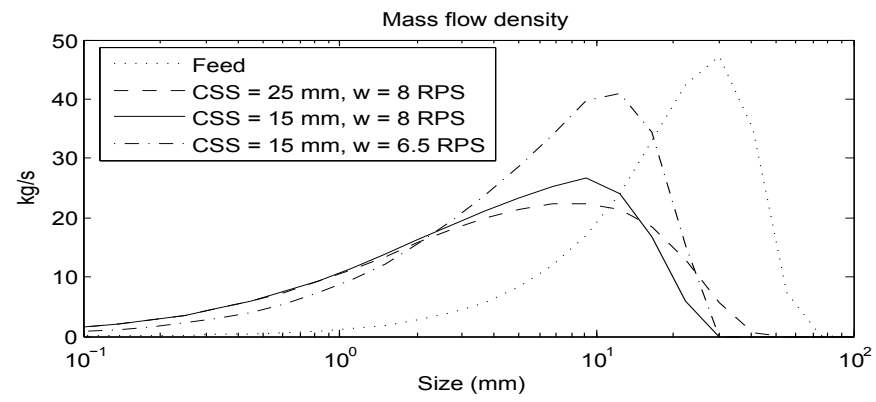

Fig. 5. Mass flow density of the feed for $D_{63}=30 \mathrm{~mm}$ and rotational speed $6 s^{-1}$. Product flow density in steady state for different values of CSS and rotational speed.

\section{CONCLUSIONS AND FUTURE WORK}

A generic distributed parameter model for cone crushers has been developed. Simulations with specific model assumptions have shown that this model exhibits expected behavior, in terms of response to changes in feed size distribution, as well as the manipulated inputs, i.e. closed side setting and rotational velocity. In particular, the model points out the rotational speed as a potentially valuable variable for control of the crusher.

Some issues remain before this model can be used for closed loop control of a cone crusher. Different assumptions on selection, classification, and breakage functions should be tested and parameter values for these be selected based on measurement data.

A suitable criterion for control design is likely to include minimizing the power draw of the crusher under some boundary condition on product flow and size distribution. Modeling the dependence of the power draw on the manipulated variables and the size distribution and hardness of the crusher feed is therefore a necessary step. For this, ideas from Herbst and Oblad (1985) may be used, where different hardness of the feed is modelled as parallell streams of ore, each one with a fixed hardness. Combining different proportions of these streams will then enable an arbitrary hardness to be modelled.

\section{ACKNOWLEDGEMENTS}

Financing of this research from the Hjalmar Lundbohm research center funded by the Swedish iron ore company LKAB is greatly appreciated. The author also wishes to thank Stefan Rönnbäck at Optimation AB for sharing his valuable insights on the crushing process.

\section{REFERENCES}

Ding, L., Gustafsson, T., and Johansson, A. (2007). Model parameter estimation of simplified linear models for a continuous paper pulp digester. Journal of Process Control, 17(2), 115-127.

Evertsson, C.M. (1999). Modelling of flow in cone crushers. Minerals Engineering, 12, 1479-1499.

Herbst, J.A. (1979). Rate processes in multiparticle metallurgical systems. In H.Y. Sohn and M.E. Wadsworth (eds.), Rate processes of extractive metallurgy, chapter 2. Plenum Press.

Herbst, J.A. and Oblad, A.E. (1985). Modern control theory applied to crushing. Part 1: Development of a dynamic model for a cone crusher and optimal estimation of crusher operating variables. In IFAC Automation for Mineral Resource Development, Queensland, Australia, 301-307.

Kapur, P.C. (1971). The energy-size reduction relationships in comminution of solids. Chemical Engineering Science, 26, 11-16.

King, R.P. (2001). Modeling $\&$ simulation of mineral processing systems. Butterworth-Heinemann.

Lindqvist, M. and Evertsson, C.M. (2004). Improved flow- and pressure model for cone crushers. Minerals Engineering, 17, 1217-1225.

Lütkepohl, H. (1996). Handbook of Matrices. John Wiley \& Sons.

Lynch, A.J. (1977). Mineral crushing and grinding circuits. Elsevier Scientific Publishing Company.

Thurley, M.J. and Ng, K.C. (2008). Identification and sizing of the entirely visible rocks from a $3 \mathrm{D}$ surface data segmentation of laboratory rock piles. Computer Vision and Image Understanding, 111, 170-178. 